\title{
An Improved T/2 Fractionally Spaced Blind Equalization Algorithm with Coordinate Transform
}

\author{
Xiaoping Jiang, Hao Ding*, Hua Zhang and Chenghua Li \\ College of Electronics and Information Engineering, Hubei Key Laboratory of \\ Intelligent Wireless Communications, South-Central University for Nationalities, \\ Wuhan 430074,China \\ arkage@qq.com
}

\begin{abstract}
To address the slow convergence and large residual error problems in the $T / 2$ fractionally spaced equalization with constant modulus algorithm (T/2-FSE-CMA) for high-order QAM signal in wireless communication, we analyze the distribution characters of 16QAM signal constellations and propose an improved T/2, fractionally spaced blind equalization with coordinate transform and constant modulus algorithm (T/2-FSE-RCTCMA). In this algorithm, we equalize the real and imaginary parts of the input signal in the fractionally spaced equalizen separately. By using the coordinate transform the output signals are mapped into the same circle and the error function of constant modulus is achieved. Using the error function tô adjust the weight vector of each sub-channel in the fractionally spaced equalizer, we may avoid the miss-detection caused by the T/2 FSE-CMA algorithm in muti-modulus high-order QAM signal equalization. The simulation results in wireless chamel show that compared with T/2-FSE-CTCMA and T/2- FSE-CMA, the proposed atgorithm has a faster convergence speed and a smaller residual error.
\end{abstract}

Keywords: Blind Equalization, Underwater Acoustic Channel, Fractional Spaced, Coordinates Transform

\section{Introduction}

In wireless communication, the blind equalization technique without training sequences is one of the main methods to remove Inter-Symbol Interference (ISI) [1]. The baud-spaced constantmodulus equalizer [2] has a simple structure. But its convergence speed is slow, an the steady-state error is large. The Fractionally spaced Equalizer (FSE), whose tap space is the fraction of baud space, has advantages convergence speed and steady-state error in constant modulus signal equalization. H;owever, for the high order QAM signal (16QAM) distributed on several known radius circles, the signal modulus is not constant. In this case, using the FSE-CMA for equalization will cause large mean square-errors and may not remove ISI thoroughly.

In this paper, based on the analysis of 16QAM signal constellation distribution characters and $\mathrm{T} / 2$ fractionally spaced equalizer, the real and imaginary parts of each subchannel output signals are equalized separately. The coordinates transform is performed on each equalizer output channel [6], and the error function after coordinates transform is achieved. The cost function is defined using this error function, using the cost minimization method we can get the weight vector updating equation of the $T / 2$ fractionally spaced equalizer. We then propose an improved $T / 2$ fractionally spaced coordinate transform blind equalization algorithm (T/2-FSE-RCTCMA). Compared with T/2-FSE-CTCMA and T/2-FSE-CMA, the equalization ability in wireless channel has improved significantly. 


\section{Fractionally Spaced Blind Equalization Algorithm}

The fractionally spaced equalizer is based on the baud spaced equalizer, and it oversamples the channel output signal at a sampling rate larger than the baud rate. Studies show that fractionally spaced equalizer is equal to multi-path system model [7]. As shown in Figure 1, the system input and output have the same sampling rate.

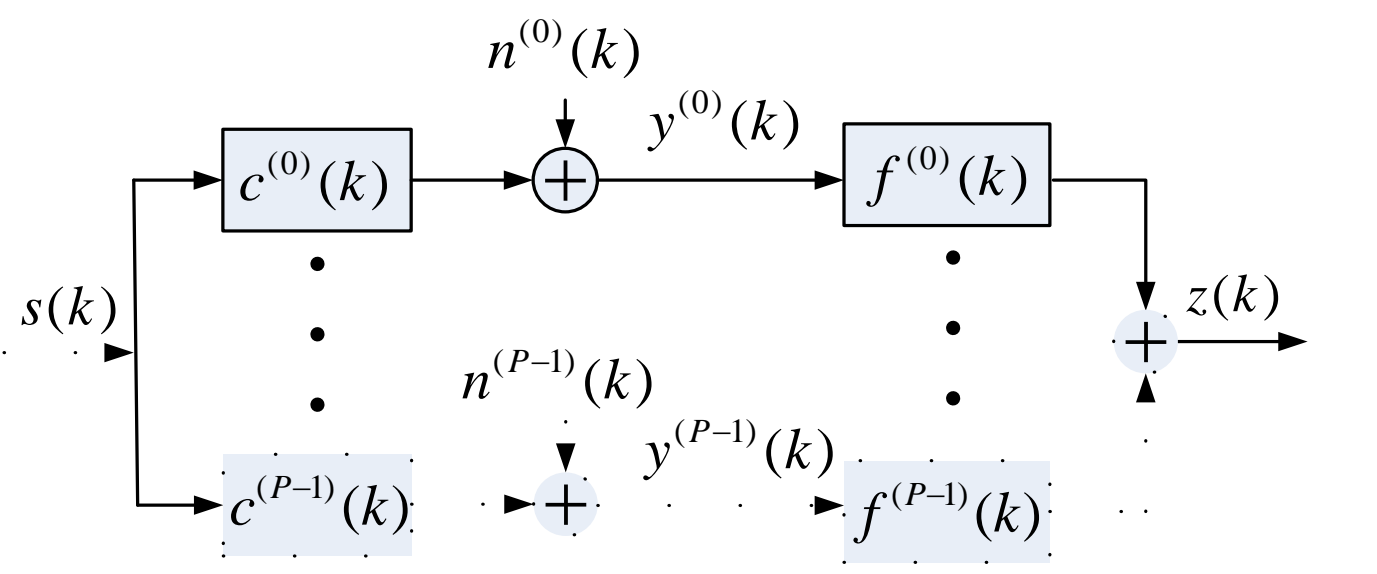

Figure 1. Structure of Fractionally Spaced Blind Equalizer

In Figure $1, s(k)$ is the transmitted signal sequence with symbol duration $T$. $\boldsymbol{c}^{(i)}(k)(i=0,1 \cdots P-1)$ is the impulse response of sub-channel. $P$ is the fractionally spaced sampling factor. The impulse response of $i-$ th channel is $\boldsymbol{c}^{(i)}(k)=c[(k+1) P-i-1] . \boldsymbol{n}^{(i)}(k)$ js the additional noise in the sub-channel. $\boldsymbol{y}^{(i)}(k)$ is the input signal of blind equalizer, which sătisfies:

$$
y^{(i)}(k) \rightleftharpoons \sum_{j=0}^{N_{c}-1} s(j) \cdot e^{(i)}(j)+n^{(i)}(k)
$$

where $N_{c}$ is the length of bat spaced channel impulse response.

$f^{(i)}(k)$ is the werght vector of equalizer, it satisfies:

$$
\boldsymbol{f}^{(i)}(k+1)=(k)+\mu z^{(i)}(k) e(k) \boldsymbol{y}^{(i)^{*}}(k)(i=0, \cdots P-1)
$$

where $\mu$ is the step length, $e(n)=R_{2}-|z(k)|^{2}$ denotes the error, and the signal modulus is $R_{2}=\left\{|s(k)|^{4}\right\} / \mathrm{E}\left\{|s(k)|^{2}\right\}$.

The output of the equalizer is:

$$
\begin{aligned}
z(k) & =\sum_{i=0}^{P-1} \boldsymbol{f}^{(i)}(k) * \boldsymbol{y}^{(P-i-1)}(k) \\
& =\sum_{i=0}^{P-1} \boldsymbol{f}^{(i)}(k) *\left[\boldsymbol{s}(k) * \boldsymbol{c}^{(P-i-1)}(k)+\boldsymbol{n}^{(P-i-1)}(k)\right]
\end{aligned}
$$

The fractionally spaced blind equalization algorithm (T/2- FSE-CMA) only fits the constant modulus signal, and it may bring a large mean square error for multi-modulus signal equalization. 


\section{Improved T/2 Fractionally Spaced Coordinates Transform Blind Equalization Algorithm}

When the transmitted signal is high-order signal, to improve the equalization result of fractionally spaced blind equalization algorithm, we make two modifications on the equalizer in Figure 1. First, equalize the real and imaginary parts of the input signal separately. It is equal to perform equalization on real signal, compared with complex signal, the calculation cost is decreased. Second, the coordinates transform is performed on the real and imaginary parts of the output signal separately, and two error functions after coordinates transform are achieved. The cost function is defined using the error functions, using the cost minimization method we can get the weight vector updating equation. The principles of the improved algorithm are shown in Figure 2. The proposed algorithm improves the equalization ability of multi-modulus signals and it also brings a faster convergence speed and a smaller steady-state error. It compensates the drawbacks of fractionally spaced constant modulus equalizer in multi-modulus signal equation

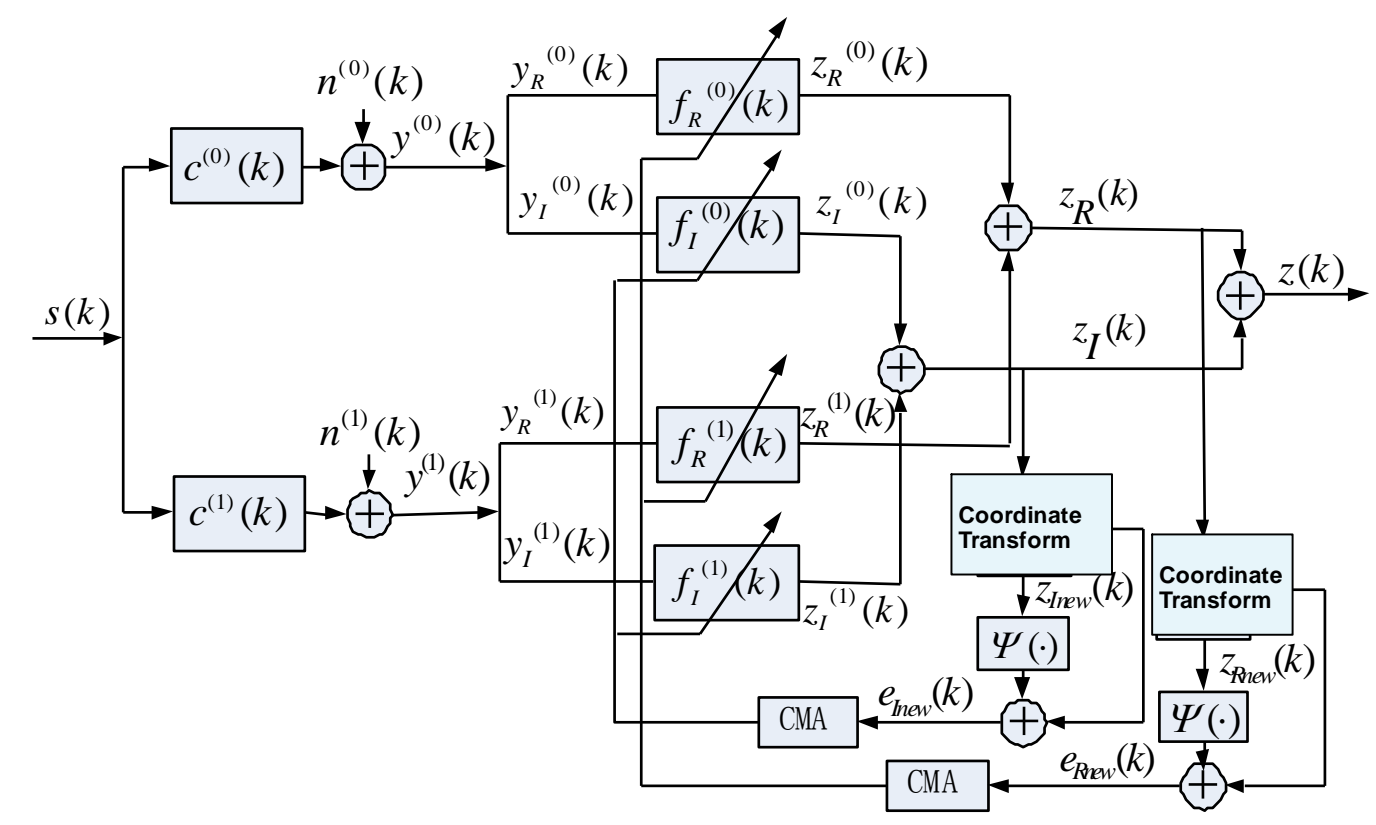

Figure 2. An Improved T/2 Fractionally Spaced Blind Equalization Algorithm with Coordinate Transform

For T/2 fractionality spaced equalizer, the oversampling factor is set to $P=2$. Based on the channel system model in Figure 1, the channel is divided into odd sub-channel $c^{(0)}(k)$ and even sub-channel $c^{(1)}(k)$. In Figure 2, the real part and the imaginary part of the inputsignal $y^{(0)}(k)$ and $y^{(1)}(k)$ are separated for equalization in the improved agorithm. The input signal is represented as:

$$
\begin{aligned}
& y^{(0)}(k)=y_{R}{ }^{(0)}(k)+j y_{I}{ }^{(0)}(k) \\
& y^{(1)}(k)=y_{R}{ }^{(1)}(k)+j y_{I}{ }^{(1)}(k)
\end{aligned}
$$

Weight vectors of each signal are $f_{R}^{(0)}(k), f_{I}^{(0)}(k), f_{R}^{(1)}(k)$ and $f_{I}^{(1)}(k)$. The output signals of the equalizer are: $z_{R}{ }^{(0)}(k), z_{I}{ }^{(0)}(k), z_{R}{ }^{(1)}(k)$ and $z_{I}^{(1)}(k)$. The real part of the final output signal is represented as:

$$
z_{R}(k)=z_{R}{ }^{(0)}(k)+z_{R}^{(1)}(k)
$$

The imaginary part is represented as: 


$$
z_{I}(k)=z_{I}^{(0)}(k)+z_{I}^{(1)}(k)
$$

The final output signal can be written as:

$$
z(k)=z_{R}(k)+j z_{I}(k)
$$

We introduce the coordinate transform to the equalization process, and the principle is demonstrated in Figure 3.

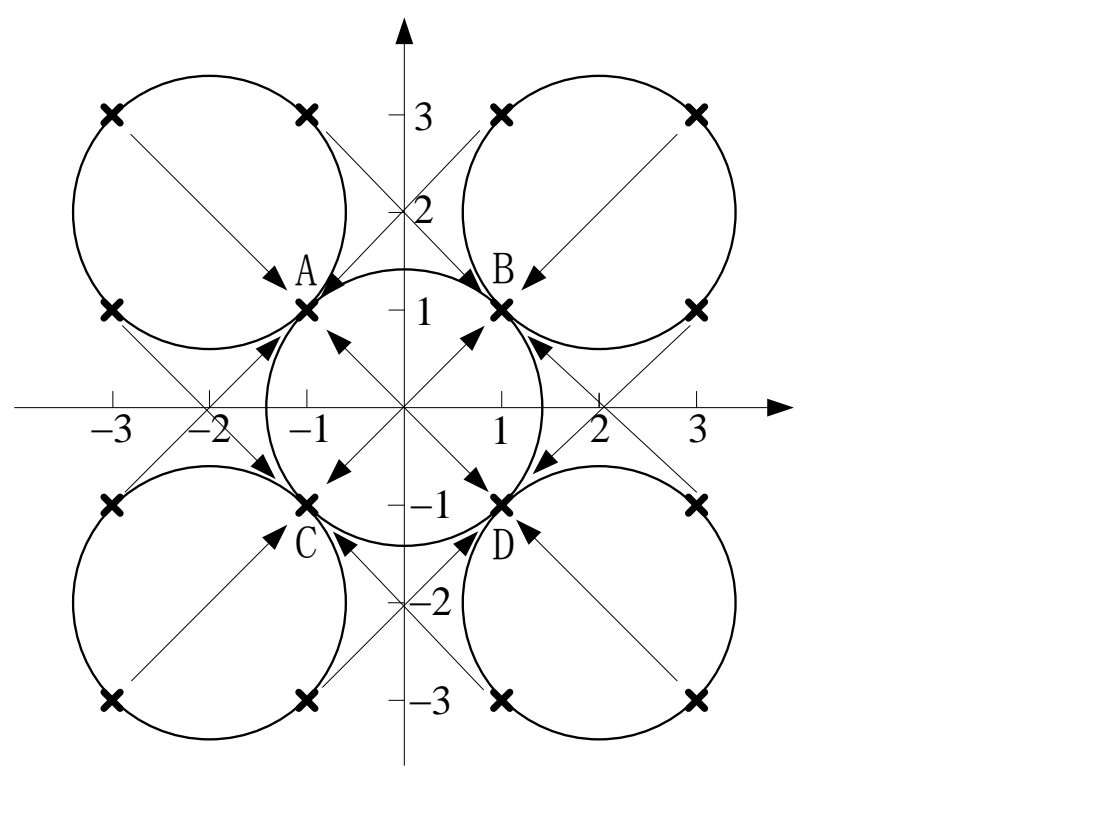

Figure 3. 16QAMCooordinate Transform Principles

In Figure 3, “ $x$ " denotes the deal 16QAM-signal points after equalization, which are distributed on four known gircles. Points A, B, C and D are four points after coordinate transform, which are located on the same circle. When the constant modulus algorithm is used for equalization, the error function is $e(n)=R_{2}-|z(k)|^{2} . R_{2}$ is a specific value, and when the signatis fully equalized, the error is not zero. The equalization result is not idea. Using coordinate transform, we can map the 16QAM signal points to points A, B, C and $\mathrm{D}$. When the signal is fully equalized, the difference between the signal modulus and the equalized signahis Zero, and the error is also zero. The equalization result is optimized.

In Figure 2, after coordinates transform, the errors of the real and imaginary parts are $e_{\text {Rnew }}(k)$ and $e_{\text {Inew }}(k)$ :

$$
\begin{gathered}
e_{\text {Rnew }}(k)=R_{\text {Rnew }}^{2}-\left|z_{\text {Rnew }}\right|^{2} \\
e_{\text {Inew }}(k)=R_{\text {Inew }}^{2}-\left|z_{\text {Inew }}\right|^{2} \\
z_{\text {Rnew }}(k)=z_{R}(k)-2 \operatorname{sign}\left[z_{R}(k)\right] \\
z_{\text {Inew }}(k)=z_{I}(k)-2 \operatorname{sign}\left[z_{I}(k)\right] \\
R_{\text {Rnew }}^{2}=\frac{E\left\{\mid\left[s_{R}(k)-2 \operatorname{sign}\left[s_{R}(k)\right]\right]\right.}{E\left\{\mid\left[s_{R}(k)-2 \operatorname{sign}\left[s_{R}(k)\right]\right]\right.} \\
R_{\text {Inew }}^{2}=\frac{E\left\{\mid\left[s_{I}(k)-2 \operatorname{sign}\left[s_{I}(k)\right]\right]\right.}{E\left\{\mid\left[s_{I}(k)-2 \operatorname{sign}\left[s_{I}(k)\right]\right]\right.}
\end{gathered}
$$

The weigh vector updating equation is: 
The output of the equalizer is:

$$
\begin{gathered}
\boldsymbol{f}_{R}^{(i)}(k+1)=\boldsymbol{f}_{R}^{(i)}(k)+\mu z_{R}^{(i)}(k) e_{\text {Rnew }}(k) \boldsymbol{y}_{R}{ }^{(i)^{*}}(k)(i=0,1) \\
\boldsymbol{f}_{I}^{(i)}(k+1)=\boldsymbol{f}_{I}^{(i)}(k)+\mu z_{I}^{(i)}(k) e_{\text {Inew }}(k) \boldsymbol{y}_{I}{ }^{(i) *}(k)(i=0,1)
\end{gathered}
$$

$$
\begin{aligned}
z(k) & =z_{R}(k)+j z_{I}(k) \\
& =\sum_{i=0}^{P-1} f_{R}{ }^{(i)}(k) \cdot y_{R}{ }^{(i)}(k)+j \sum_{i=0}^{P-1} f_{I}^{(i)}(k) \cdot y_{P}{ }^{(i)}(k)
\end{aligned}
$$

For 16QAM signals, when the channel is fully equalized, Eq. (9) equals to zero. Eq. (4) to (14) give out the improved $\mathrm{T} / 2$ fractionally spaced blind equalization with coordinate transformation. In this paper, we name the algorithm that only do the coordinate transform on the output signals T/2-FSE-CTCMA. The improved algorithm T/2-FSE-RCTCMA performs equalization on real and imaginary parts separately, which is equal to the real signal. Therefore its calculation cost is smaller than that of T/2-FSE-CTCMA. Since the improved algorithm perform coordinate transform on the real and imaginary parts of the output signal separately, the convergence speed is faster and the steady-state error is smaller, compared with T/2-FSE-CTCMA and T/2-FSE-CMA.

\section{Algorithm Performance Analysis}

\subsection{Convergence Analysis}

$\mathrm{T} / 2$ fractionally spaced equalizer adopts sampling rate of $\mathrm{T} / 2$, and it avoids the overlapping of the spectrum caused by under sampling. It also compensates the channel distortion [8]. The new algorithm performs equalization on the real and imaginary parts separately, which is equal to process reals gnal and the computational cost decreases. The signal is converted from multi-modulus to constant modulus by coordinate transform, therefore the steady-state error Cedreases and the convergence speed increases. For 16QAM, after separating the real and imagmary parts, it equals to two channels of 4PAM signals. After coordinate transform, it is converted to 2PAM, and the updating of weigh coefficients is faster. When the channel is fully equalized, error function converges to zero. Therefore, the equatization speed is significantly faster, the convergence speed is also faster, and the steady-state error is smaller. We verified the advantages of this new algorithm in wireless simulation experiments.

\subsection{Analysis of Computation Load}

In each iteration of weight vectors in T/2-FSE-CMA, there are $\left(N_{f} / 2\right) * 4$ multiplications and $\left(N_{f} / 2\right) * 3+\left[\left(N_{f} / 2\right)-1\right]$ additions for each channel of signal. In T/2 FSE-CTCMA, where the real and imaginary parts are separated, there are $N_{f}-2$ multiplications and $\left(N_{f} / 2\right)-1$ additions for the real parts in each iteration. The total computation load for one channel of signal includes $N_{f}$ multiplications and $N_{f}-2$ additions. Based on the above analysis, the computation load of T/2-FSE-CTCMA is almost half of the T/2-FSE-CMA. Meanwhile, the simulation results also indicate that the equalization performance of the proposed algorithm is better than that of T/2-FSE-CMA. 


\section{Simulation Experiments}

In order to verify the efficiency of T/2-FSE-RCTCMA algorithm, we compared with T/2-FSE-CTCMA and T/2-FSE-CMA in wireless channel simulation experiments.

[Experiment 1] Use the mixed phase wireless channel [9].

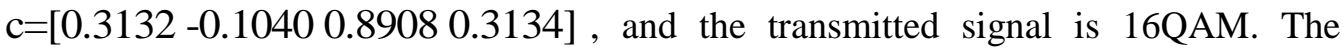
weight vector length of the equalizer is $32, \mathrm{SNR}$ is $25 \mathrm{~dB}$. The weight vector length of each sub-channel is 16 , and weight vector is initialized as central tap. The steps of three algorithms $\left(\mu_{\mathrm{T} / 2-\mathrm{FSE}}, \mu_{\mathrm{T} / 2-\mathrm{FSE}-\mathrm{CTCMA}}, \mu_{\mathrm{T} / 2-\mathrm{FSE}-\mathrm{RCTCMA}}\right)$ are set to value 0.000006 , 0.00003 , and 0.0009 respectively. The 5000 Monte Carlo simulation results are shown in Figure 4.

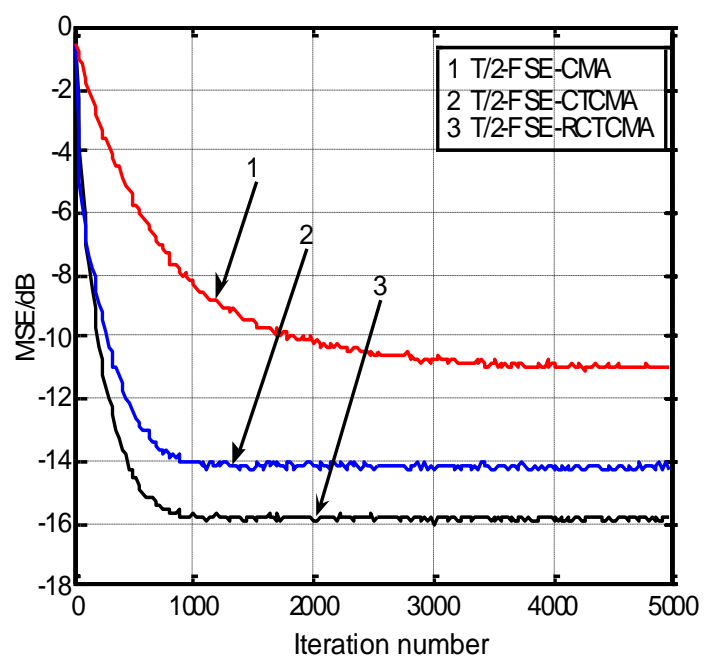

(a) Error curves

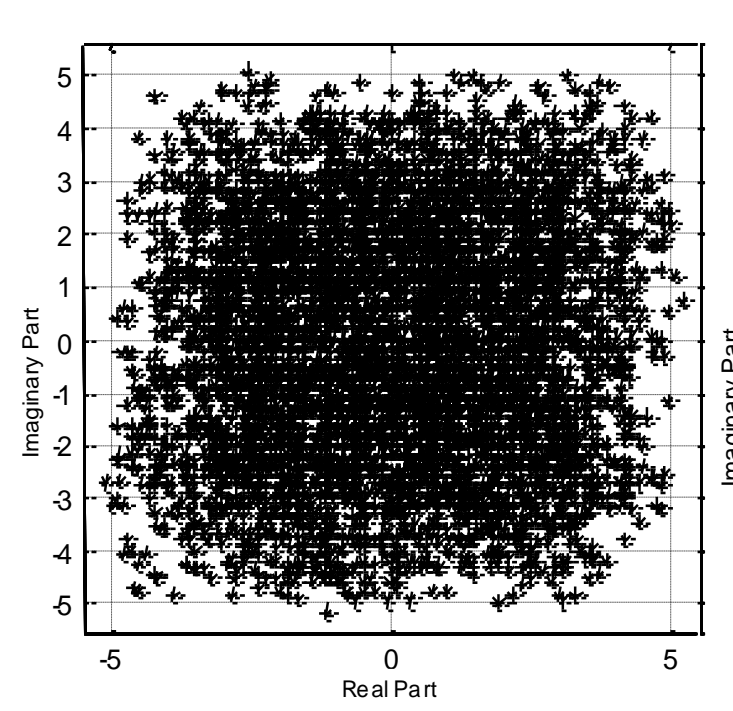

(c) Input signal of equalizer

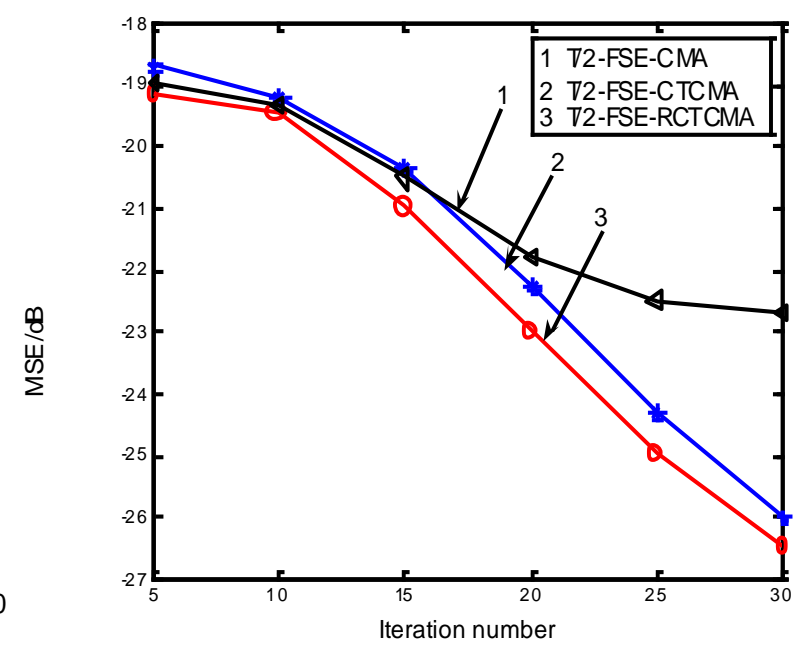

(b) Root mean square error curve

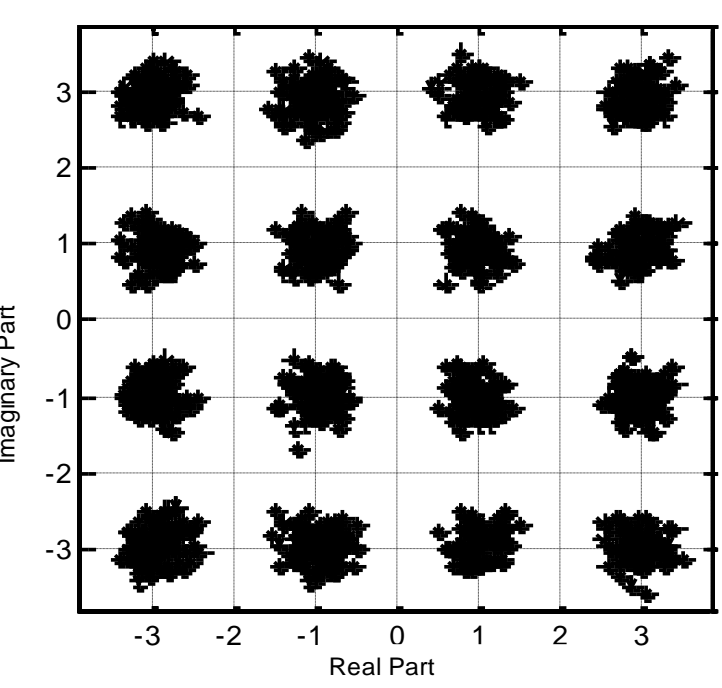

(d) T/2-FSE-CMA constellation 


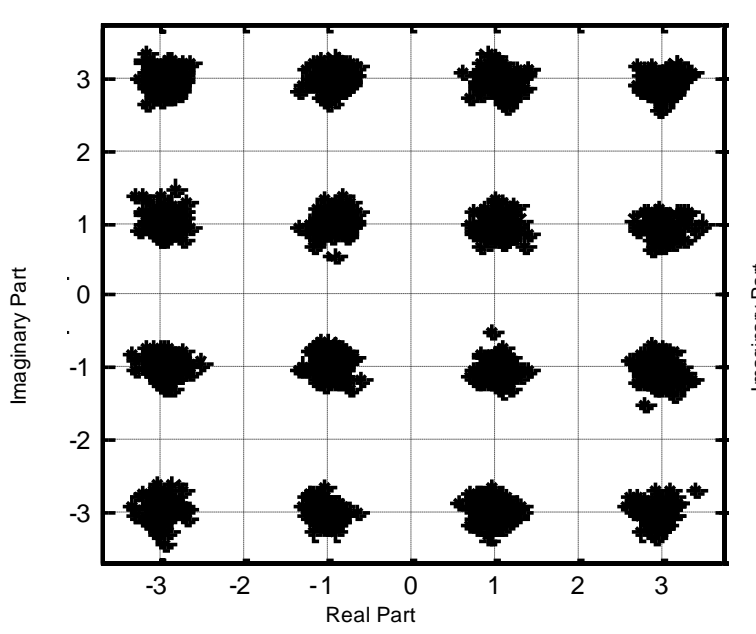

(e) T/2-FSE-CTCMA constellation

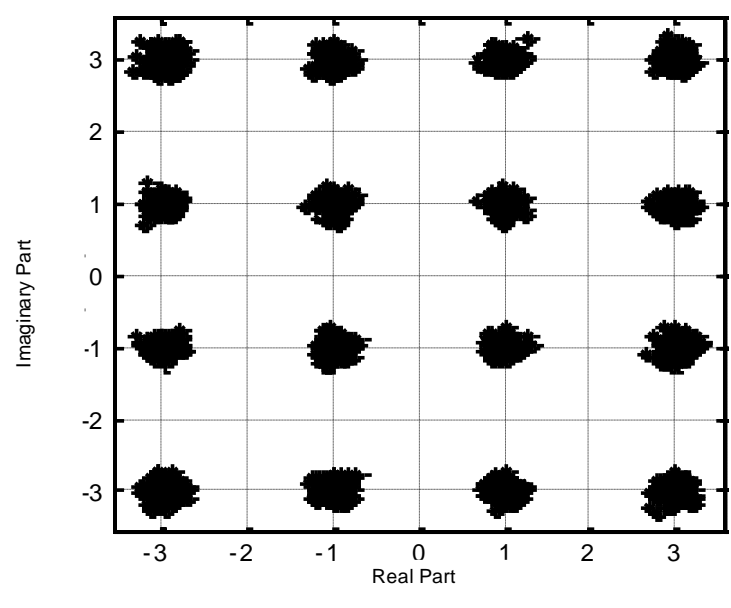

(f) T/2-FSE-RCTCMA constellation

\section{Figure 4. Simulation Results}

In Figure 4(a), the simulation results show that the steady-state error of T/2-FSERCTCMA is $2 \mathrm{~dB}$ smaller than that of T/2-FSE-CTCMA, and $5 \mathrm{~dB}$ smaller than that of T/2-FSE-CMA. In convergence speed T/2-FSE-RCTCMA is the fastest one, 2000 step ahead of T/2-FSE-CMA. In Figure 4(b), under various SNRs, the root mean square error of T/2-FSE-RCTCMA is the smallest. The constellations in Figure 4 (d), (e) and (f) further proved that T/2-FSE-RCTCMA Outperforms T/2 FSE-CMA. The signal constellation of T/2-FSE-RCTCMA is elearer and brings higher anti-ISI ability.

\section{Conclusion}

In this paper we propose the $T / 2$ fractionally spaced blind equalization algorithm with coordinate transform (T/2-ESE-RCTCMA). Its computation load is reduced by almost fifty percent and it shows elear advantage for 16QAM signal equalization. The wireless channel simulation results show that: compared with T/2-FSE-CTCMA and T/2-FSECMA, the algorithm bas a faste convergence speed and smaller residual error. The effect on its constellation is quite obvious. Therefore, this algorithm may efficiently removing ISI and recover ignal in real tome.

\section{Acknowledgements}

This work was supported by the General Program of the Natural Science Fund of Hubei Province (2014CFB290) and the Key Project of the Fundamental Research Funds for the Central Universities (CZQ14001 and CZW15043). 


\section{References}

[1] C. R. Johnson, P. Schniter Jr and I. Fijalkow, "The core of FSE-CMA behavior theory", Simon Haykin.Unsupervised Adapitive Filtering Volume II :Blind Deconvolution, New York: Wiley, (2000), pp. 12-112.

[2] W. Rao, K.-M. Yuan, Y.-C. Guo and C.Yang, "A Simple Constant Modulus Algorithm for Blind Equalizer Suitable for 16-QAM Signal”, International Conference on Signal Processing Proceedings, (2008), pp. 1963-1966

[3] O. Dabeer and E. Masry, "Convergence analysis of the constant modulus algorithm", IEEE Transactions on Information Theory, vol. 49, no. 6, (2003), pp. 1447-1464.

[4] L. Zhou, J. Li and G. Zhang, "Novel DWPM system based on fractionally spaced equalizers and the maximum likelihood algorithm", Journal of Xidian University (Natural Science Edition), (2006), vol. 33, no. 4, pp. 509-513.

[5] K. C.H. Ren and Q. Tu, "A method of improving frequency resolution of modified covariance spectrum estimation and its abilities to adapt noise using multistage wavelet de-composition", Journa of Electronic Measurement and Instrument, vol. 20, no. 4, (2006), pp. 15-21.

[6] W. Rao, K.-M. Yuan, Y.-C. Guo and C.Yang, "A Simple Constant Modulus Algorithm For Blind" Equalizer Suitable for 16-QAM Signal”, International Conference on Signal Processing Proceedings, (2008), pp. 1963-1966.

[7] B. Wu, Q. Chen and S. Cheng, "Performance of wavelet transform domain adaptive equalizers , Journal of Southeast University (English Edition), vol. 18, no. 1, (2002), pp. 13 18

[8] T. Cooklev, "An Efficient Architecture for Orthogonal Wavelet Transforms", IEEE Signal Processing Letters(S1070-9980), vol. 13, no. 2, (2006), pp. 77 79.

[9] O. Dabeer and E. Masry, "Convergence analysis of the constant modulus algorithm", IEEE Transactions on Information Theory, (2003), vol. 49, no. 6, pp. 1447-(464

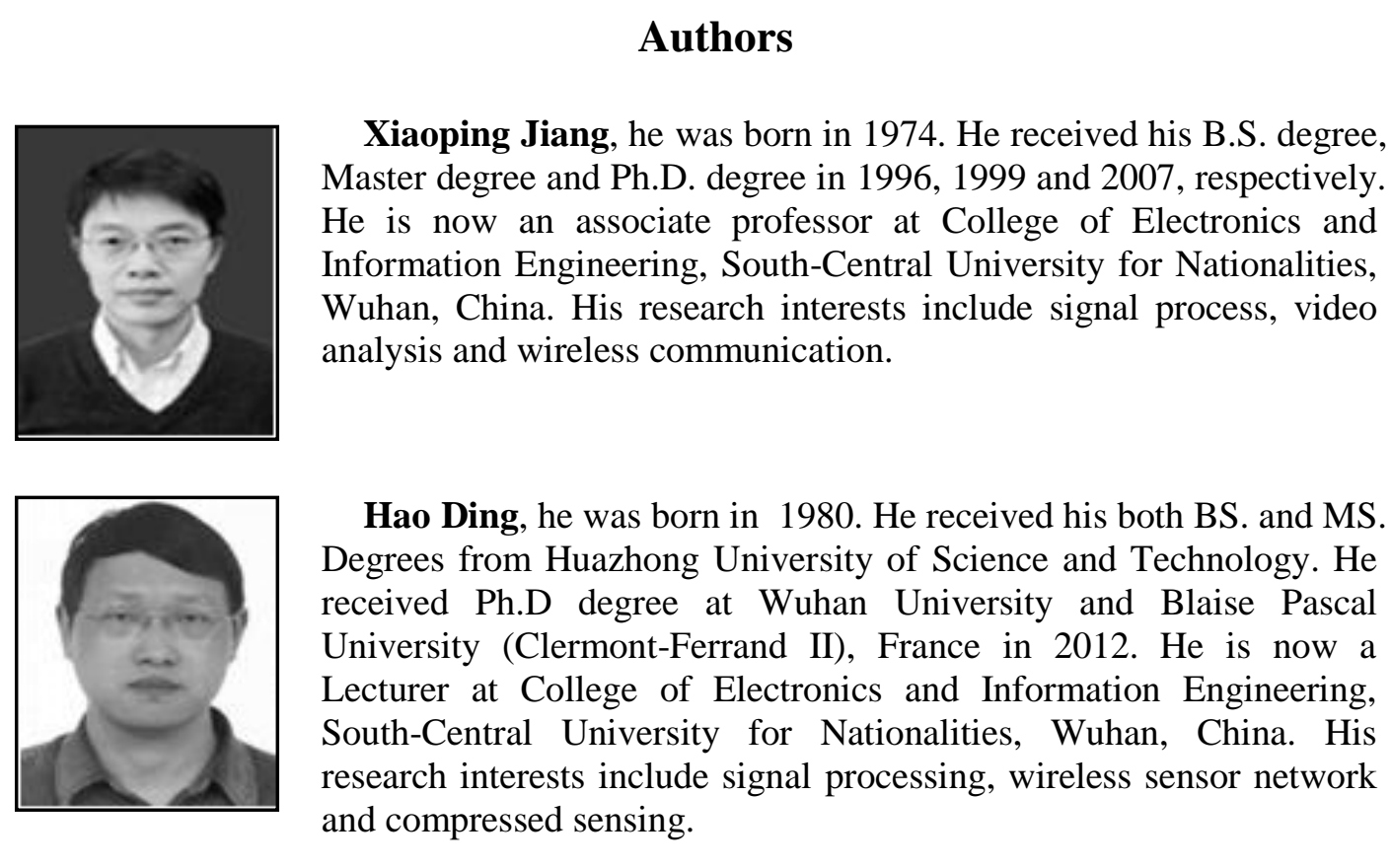

Hua Zhang, he was born in 1972. He received his B.S. degree, Master degree and Ph.D. degree in 1995, 2002 and 2011, respectively. He is now a Lecturer at College of Electronics and Information Engineering, South-Central University for Nationalities, Wuhan, China. His research interests include intelligent control, image analysis and celestial navigation. 


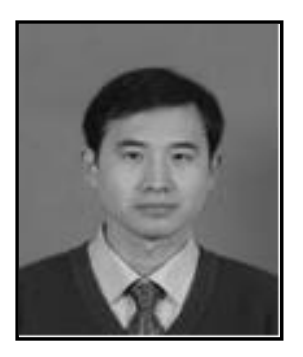

Chenghua Li, he was born in 1972. He received his B.S. degree, Master degree and Ph.D. degree in 1994, 2004 and 2008, respectively. $\mathrm{He}$ is now a associate professor at College of Electronics and Information Engineering, South-Central University for Nationalities, Wuhan, China. His research interests include cloud computing, image analysis and pattern recognition

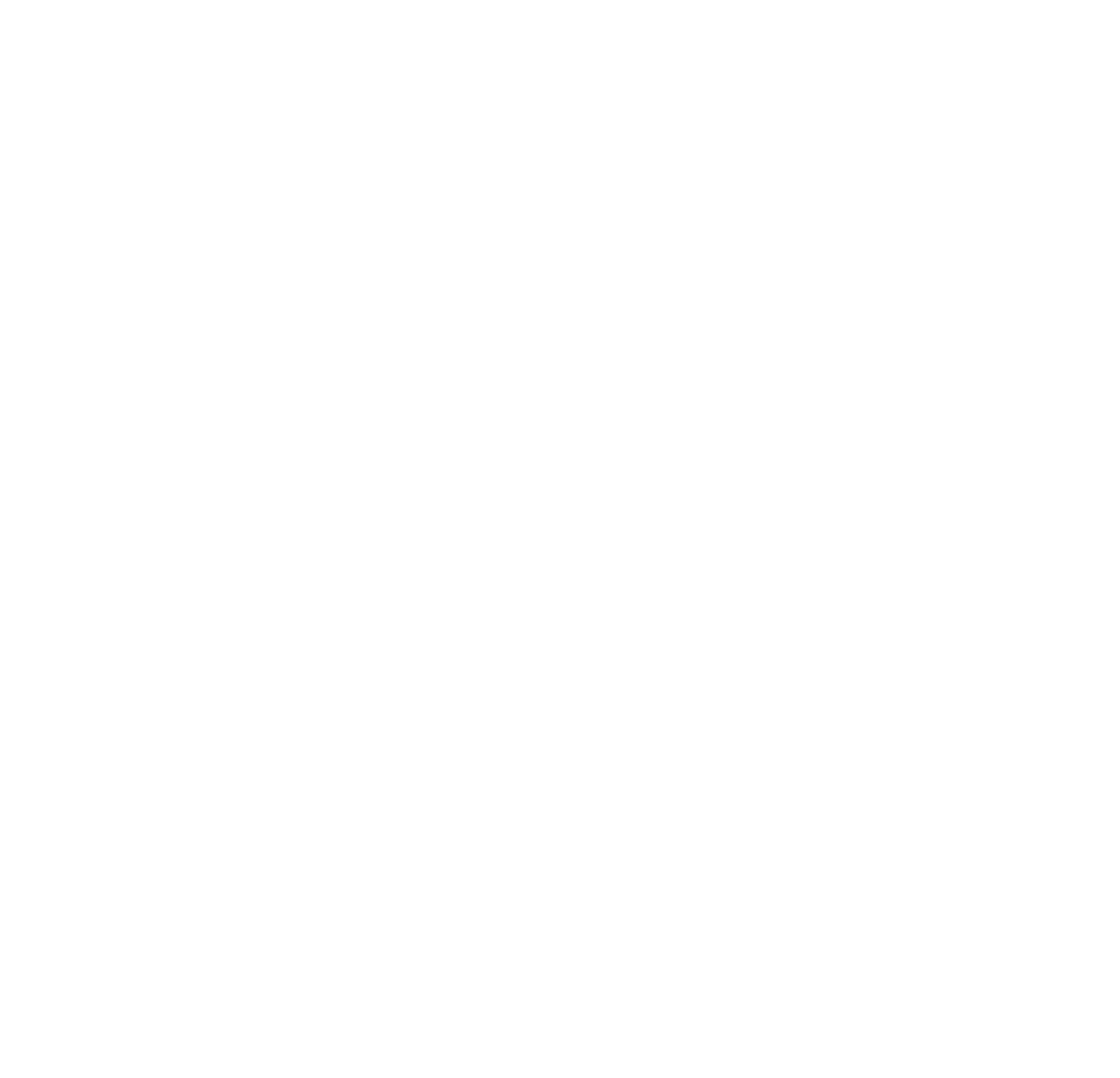


International Journal of Hybrid Information Technology

Vol. 9, No.12 (2016)

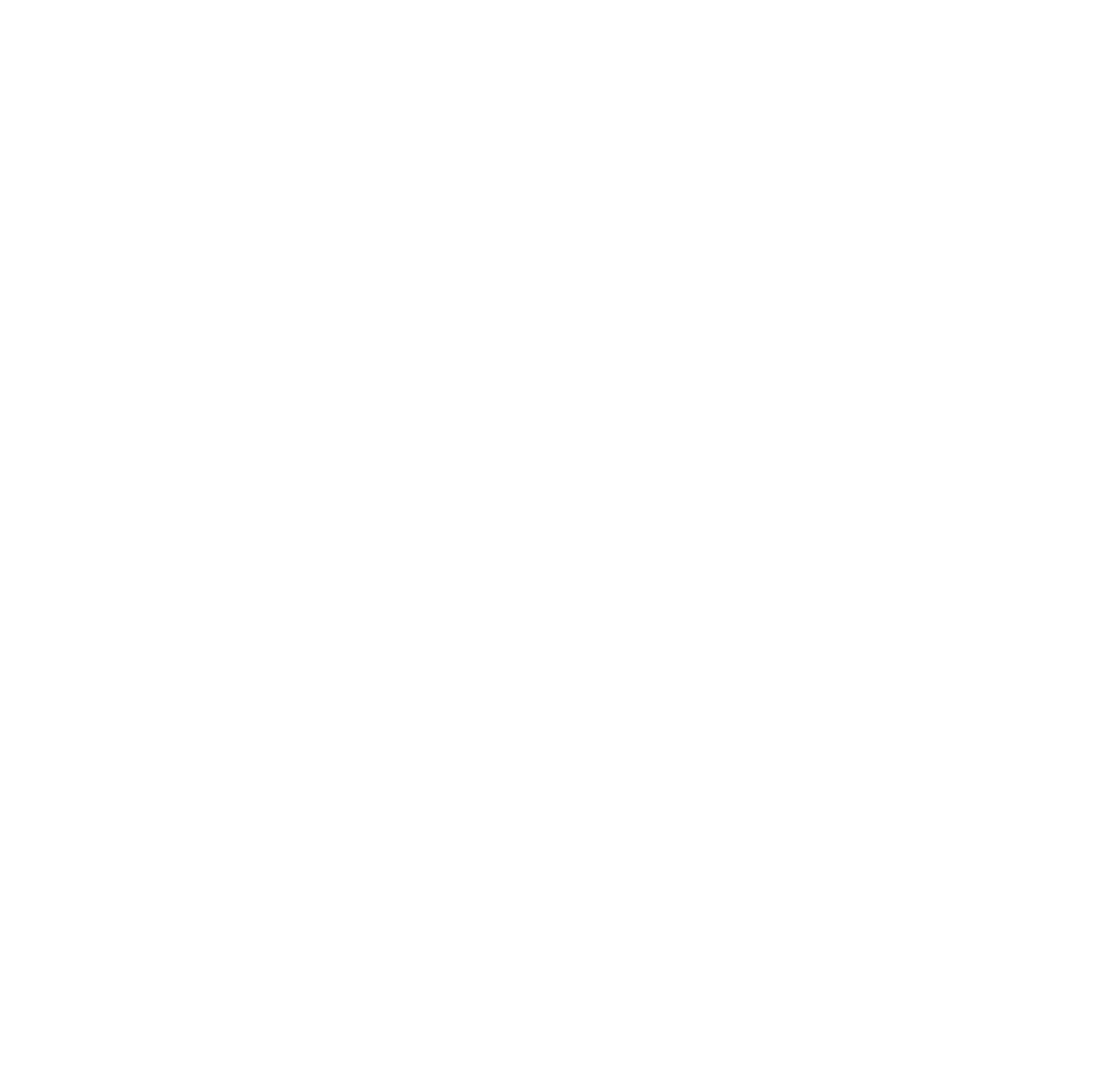

